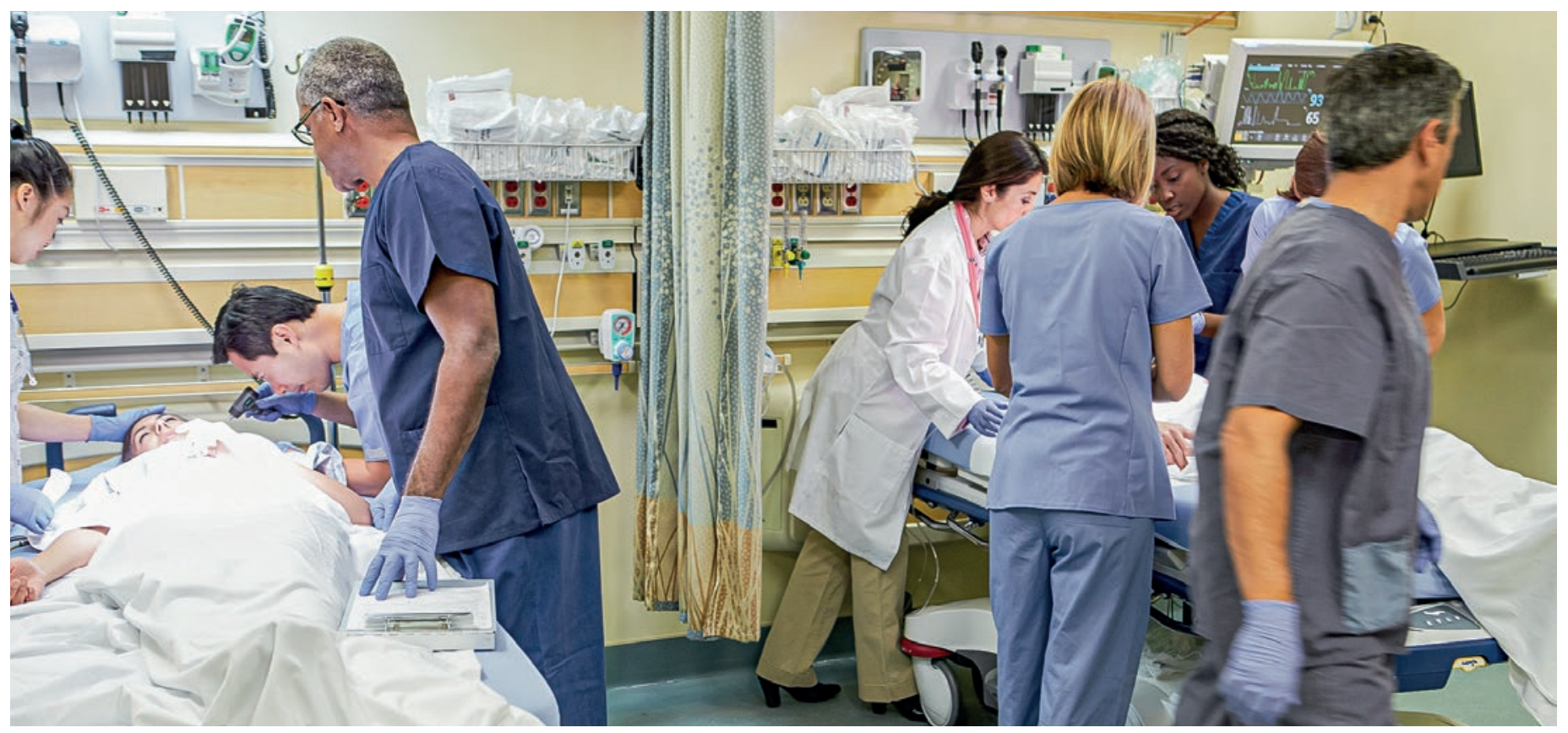

\title{
Un bon départ pour un avenir commun
}

\section{Cinzia Zeltner}

Collaboratrice scientifique, responsable adjointe de la section Développement professions de la santé, Office fédéral de la santé publique (OFSP)

Le programme de promotion «Interprofessionnalité» s'est achevé il y a un an environ. Que nous a-t-il appris? Comment l'Office fédéral de la santé publique (OFSP) a-t-il poursuivi le travail sur le sujet? Et surtout: quelles sont les prochaines étapes?

L'un des principaux objectifs du programme de promotion de l'interprofessionnalité était la pertinence de ses produits pour la pratique. Les résultats des projets de recherche devaient non seulement fournir une base scientifique, mais également être intégrés dans la vie professionnelle et éducative quotidienne. Cet objectif a été largement atteint. La boîte à outils pour la santé psychique «Take Care», élaborée par l'Université des Sciences Appliquées de Zurich (ZHAW), qui comporte des informations pour les professionnels, une brochure pour les adolescents et un flyer pour les enfants et les personnes en charge de l'éducation de ces derniers, a par exemple connu un tel succès que le tirage original a déjà dû être réimprimé.

L'instrument suisse pour l'évaluation de l'interprofessionnalité (ISEIP) mis au point et validé par l'Institut d'enseignement médical (IML) de Berne et la Haute Ecole spécialisée bernoise (BFH) a lui aussi déjà trouvé son application pratique. L'ISEIP permet aux institutions de mesurer le niveau d'interprofessionnalité de leur environnement de travail. Il comprend des questionnaires pour le personnel, la direction et les patientes et patients.

\section{Modèles de bonnes pratiques peu connus}

Outre les projets de recherche, le programme de promotion a également servi à financer un catalogue des modèles de bonnes pratiques. L'idée était simple: l'interprofessionnalité est déjà une réalité vécue un peu partout en Suisse, mais ces modèles ne sont souvent connus qu'au niveau régional. Le catalogue a pour but 
de les réunir, de leur donner une visibilité nationale et de favoriser les échanges entre les personnes intéressées. Certains de ces modèles ont été décrits en détail dans deux brochures afin de fournir au public concerné un aperçu approfondi de leur fonctionnement.

Le catalogue mérite que l'on y jette un coup d'œil, parce qu'il couvre un large éventail de sujets, de cadres de travail et de groupes professionnels. Les modèles recueillis visent pour beaucoup à optimiser la collaboration entre professionnels afin qu'ils puissent se concentrer davantage sur leurs activités principales et

\section{L'interprofessionnalité est une réalité vécue en Suisse, mais les modèles de bonnes pratiques ne sont souvent connus qu'au niveau régional.}

améliorer la qualité des soins. Le projet d'intervention d'infirmières spécialisées diplômées (advanced practice nurses) dans le cabinet du médecin de famille de Bürglen, soutenu par le canton d'Uri et dont l'évaluation scientifique a été réalisée par le Zentrum für Hausarztmedizin und Community Care de l'Université de Lucerne, en est un exemple. Le modèle du service psychiatrique de l'Emmental montre lui aussi comment la gestion interprofessionnelle des cas peut optimiser les processus et les soins.

D'autres entrées concernent la formation et l'enseignement, par exemple les modules de discussion interprofessionnelle de cas de l'Hôpital universitaire de Zurich suivis par les étudiants en médecine, en soins infirmiers, en obstétrique, en physiothérapie, en ergothérapie, en diététique et en travail social. Il existe aussi des

\section{Volets thématiques communs}

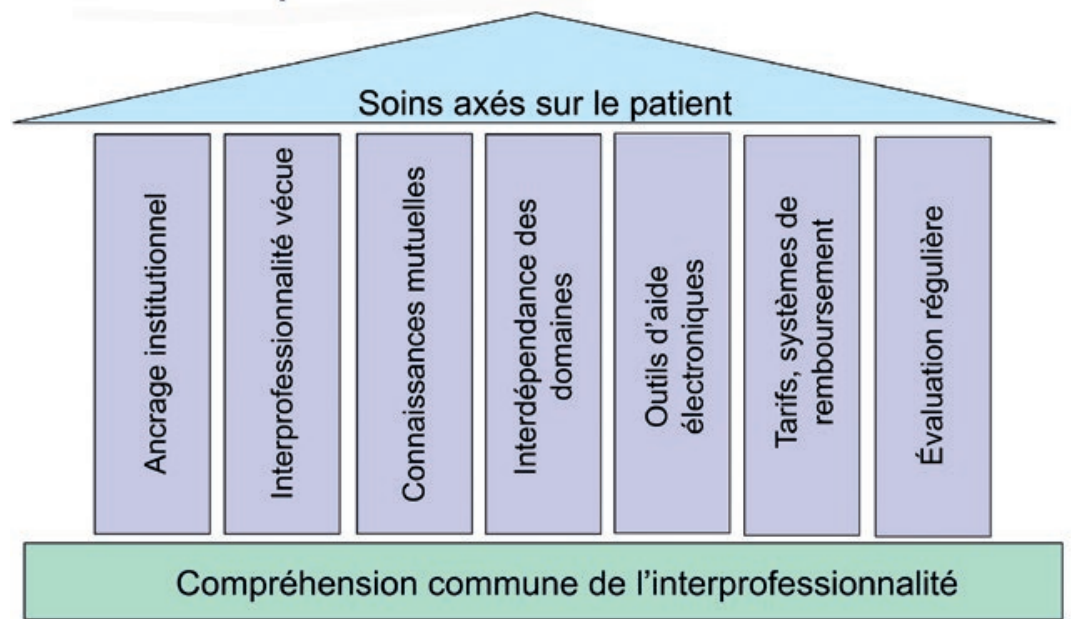

Futurs champs d'action sur la base de sept volets thématiques. Source: OFSP 2020 entrées concernant des outils, tels que «SisCare», que les médecins de famille, les infirmières et infirmiers et les pharmaciennes et pharmaciens peuvent utiliser pour optimiser le traitement des malades chroniques. Le catalogue est un engagement à long terme de l'OFSP. Il est complété au fur et à mesure et sera migré à la mi2022 vers un site Internet indépendant, qui comprendra des exemples de bonnes pratiques tirés de divers domaines de la santé et proposera aux personnes intéressées des aides pour réaliser leurs propres projets.

\section{Mesures supplémentaires nécessaires}

Même si le programme de promotion a permis de combler de nombreuses lacunes dans la recherche et si la transposition dans la pratique s'est globalement bien passée, les travaux ont néanmoins montré que des mesures supplémentaires sont nécessaires. Elles ont été rassemblées dans les quatre Policy Briefs «Soins ambulatoires», "Soins stationnaires», "Jonction entre soins psychiques et somatiques» et "Formation». Elles peuvent être divisées en sept volets thématiques (voir graphique). Les mesures à venir devraient donc promouvoir l'ancrage institutionnel de l'interprofessionnalité, l'interprofessionnalité vécue par la direction et les cadres, les connaissances mutuelles des rôles et des compétences, et l'interdépendance des différents domaines du secteur de la santé. Mais elles devraient aussi améliorer les conditions-cadres, comme le fonctionnement des outils électroniques, jugé insatisfaisant, ou les tarifs et systèmes de remboursement. L'évaluation régulière de la mise en œuvre de l'interprofessionnalité, c'est-à-dire l'utilisation d'outils tels que l'ISEIP, fait également partie des champs d'action identifiés.

\section{Parvenir à une compréhension commune}

Tous les champs thématiques ont un but commun: améliorer la prise en charge des patientes et patients. Pour atteindre cet objectif, les mesures à venir doivent reposer sur la même base, à savoir sur une compréhension commune de l'interprofessionnalité. Au fil du programme de promotion, il est apparu que ce n'est pas encore le cas en Suisse. C'est pourquoi, au début de l'année 2021, l'OFSP a chargé le bureau de conseil Vatter de dresser un état des lieux de la définition de l'interprofessionnalité dans la littérature et de l'utilisation de ce terme en Suisse.

Celui-ci a révélé un décalage entre la théorie et la pratique: si, dans la théorie, l'interprofessionnalité est assez clairement délimitée par rapport à d'autres concepts comme la délégation de tâches (task shifting), 
dans la pratique, le terme est souvent utilisé comme synonyme d'autres notions. Cet aspect est important pour la suite: l'OFSP doit en effet savoir s'il convient, lors de la promotion de nouveaux projets, d'appliquer la définition scientifique ou de conserver l'interprétation plus familière. Dans le cadre de son processus stratégique, l'OFSP va formuler une définition de l'interprofessionnalité valable pour la Suisse et en déduire des mesures.

\section{Promouvoir la mise en réseau}

Il est également ressorti des Policy Briefs que l'OFSP doit constituer un réseau pour renforcer la coordination entre les acteurs. L'office a donc mené divers entretiens avec les parties prenantes au printemps 2021 pour clarifier la manière dont un tel réseau pourrait

\section{La mise en réseau des acteurs doit être renfor- cée. L'OFSP est prêt à en assurer la conduite dans le domaine de la formation.}

être organisé. Ceux-ci ont confirmé que dans la formation, en particulier, il n'existe que des structures informelles et souvent régionales. Ils ont aussi montré que les parties prenantes rejetaient clairement l'idée d'un réseau supplémentaire et supérieur. Elles estimaient bien plus judicieux de mettre davantage en réseau les structures existantes et souhaitaient majoritairement que cette tâche soit confiée à la Confédération.

L'OFSP est prêt à diriger le renforcement de la coordination entre les réseaux existants de formation interprofessionnelle, mettant ainsi au point des mesures possibles en milieu d'année. La planification concrète de la suite est toutefois retardée du fait que, pour décider d'un bon nombre de ces mesures, l'OFSP doit savoir s'il disposera ou non dans les prochaines années de fonds supplémentaires pour soutenir l'interprofessionnalité sous la forme d'un second programme de promotion.

\section{Un thème qui restera central}

La réponse à cette question dépend notamment de l'issue de la votation sur l'initiative sur les soins infirmiers le 28 novembre 2021. Si celle-ci est rejetée et que le contre-projet indirect entre en vigueur, l'OFSP pourra lancer un deuxième programme de promotion. En effet, le contre-projet créerait à la fois les bases légales nécessaires et mettrait à disposition des moyens financiers à hauteur de cinq millions de francs pour soutenir de tels projets. Ce ne sera en revanche pas le cas si l'initiative est acceptée. Des projets de l'envergure d'un programme de promotion ne pourraient alors pas être réalisés et les mesures envisagées pour promouvoir le Réseau Interprofessionnalité devraient également être allégées. L'OFSP se chargerait certes toujours des tâches de coordination du réseau, mais des investissements de plus grande ampleur ne seraient pas réalistes.

Quelle que soit l'issue de la votation, l'interprofessionnalité restera un thème central de la stratégie Santé2030. Les travaux évoqués pour une meilleure coordination du réseau et la migration des modèles de bonnes pratiques vers un site Internet externe en sont deux exemples. Le thème de l'interprofessionnalité pourrait en outre être régulièrement abordé en cas de révision des objectifs généraux de formation et de l'accréditation des filières de formation postgrade des professions médicales dans les années à venir. L'OFSP entend également faire de l'interprofessionnalité un thème central de la plateforme "Avenir de la formation médicale» qui travaillera l'an prochain intensivement sur l'image du médecin du futur. La prise en charge interprofessionnelle est en outre soutenue dans le cadre des mesures de maîtrise des coûts, qui envisagent de définir les réseaux de soins coordonnés en tant que fournisseurs de prestations distincts, ce qui devrait faciliter la mise en œuvre de nouveaux modèles de soins.

On saura dans quelques jours s'il sera également possible de financer des projets et des programmes interprofessionnels allant plus loin.

Informations complémentaires

- Programme de promotion «Interprofessionnalité»: www.bag. admin.ch/ppinterprof

- Instrument d'évaluation ISEIP (IML Berne, BFH): evaluation[at]iml unibe.ch (contact via e-mail)

- Boîte à outils Take Care (ZHAW): https://www.zhaw.ch/fr

- Modèles de bonnes pratiques (OFSP): www.bag.admin.ch/ modeles-interprof

Crédits photo

Monkey Business Images | Dreamstime.com

\section{Série sur l'interprofessionnalité}

La collaboration entre les spécialistes de différentes professions de la santé est considérée comme un levier important afin de relever les défis du système de santé. Où en est la Suisse dans ce domaine? Quels avantages apporte l'interprofessionnalité et quelles en sont les limites? Nous éclairons le sujet sous divers angles dans une série d'articles. 